\title{
Validation of a novel sleep-monitoring system for diagnosing obstructive sleep apnea: A comparison with polysomnography
}

\author{
LILI MENG ${ }^{1}$, HUAJUN XU ${ }^{1,2}$, JIAN GUAN ${ }^{1,2}$, HONGLIANG YI ${ }^{1,2}$, HONGMIN WU $^{1}$ and SHANKAI YIN ${ }^{1,2}$ \\ ${ }^{1}$ Department of Otolaryngology, Shanghai Jiao Tong University Affiliated Sixth People's Hospital; \\ ${ }^{2}$ Otolaryngology Institute of Shanghai Jiao Tong University, Shanghai 200233, P.R. China
}

Received June 11, 2015; Accepted March 21, 2016

DOI: $10.3892 / \mathrm{etm} .2016 .3721$

\begin{abstract}
Overnight polysomnography (PSG) is currently the gold standard for diagnosing obstructive sleep apnea (OSA); however, it is time-consuming, expensive and uncomfortable for the patient. A micromovement sensitive mattress (MSM) sleep-monitoring system was developed as an alternative to PSG, however, there has yet to be a study verifying the accuracy of diagnosing OSA with this device. Therefore, the present study assessed the validity of the MSM sleep-monitoring system. Chinese Han participants who were suspected of having OSA were recruited between June 2013 and June 2014. The MSM sleep-monitoring system and PSG were utilized simultaneously overnight on each subject. The apnea-hypopnea index (AHI) was measured by the MSM sleep-monitoring system $\left(\mathrm{AHI}_{\mathrm{MSM}}\right)$ and compared with that determined by PSG $\left(\mathrm{AHI}_{\mathrm{PSG}}\right)$, revealing a significant correlation between the two values $(\mathrm{r}=0.97, \mathrm{P}<0.001)$. Bland-Altman plots also indicated good agreement $(97 \%)$ between MSM and PSG. Using an $\mathrm{AHI}_{\mathrm{PSG}}$ cut-off of $\geq 5, \geq 15$ and $\geq 30$ events/h, the sensitivity (specificity) of detecting an $\mathrm{AHI}_{\mathrm{MSM}}$ of $\geq 5, \geq 15$, and $\geq 30$ events/h were 94.9 (100\%), 89.9 (96.9\%) and $90.3 \%$ (94.9\%), respectively. The areas under the receiver operating characteristic curve, which were used to differentiate an $\mathrm{AHI}_{\mathrm{PSG}}$ of $\geq 5, \geq 15$ and $\geq 30$ events/h in clinically diagnosed OSA, were $0.984,0.982$ and 0.980 , respectively. Thus, the MSM sleeping system may accurately diagnose OSA in the Chinese Han population. Further community-based studies with larger sample sizes are warranted to confirm the validity of this MSM sleeping system.
\end{abstract}

Correspondence to: Dr Huajun Xu or Dr Hongmin Wu, Department of Otolaryngology, Shanghai Jiao Tong University Affiliated Sixth People's Hospital, 600 Yishan Road, Shanghai 200233, P.R China E-mail: sunnydayxu2010@163.com

E-mail: wuhongmin88@126.com

Key words: micromovement sensitive mattress, polysomnography, obstructive sleep apnea

\section{Introduction}

Obstructive sleep apnea (OSA) is a chronic sleep disorder with high morbidity and mortality, affecting $9 \%$ of middle-aged women and $24 \%$ of middle-aged (30-55 years old) men. OSA increased the risk of the overall mortality by $26.2 \%$ (1). Epidemiologically, OSA is an independent risk factor for hypertension, diabetes mellitus, coronary heart disease and even mortality (2-5). Intermittent hypoxemia-induced oxidative stress and inflammation, combined with increased sympathetic activation, are potential mechanisms linking OSA and its cardiovascular or metabolic sequelae (6). In addition to the OSA-associated health consequences, OSA results in increasing social and economic burdens, including car accidents, inefficiency at work and reduced social ability (7-9). Although general practitioners are aware of OSA, there is a limited availability of efficient diagnostic methods, and a diagnosis of OSA is important, since it may be associated with other serious complications (10). The majority of undiagnosed OSA patients are not aware of the dangers caused by OSA and do not seek therapy (11).

As recommended by the American Academy of Sleep Medicine (AASM), overnight polysomnography (PSG) is the gold standard for diagnosing OSA (12). However, several limitations of PSG monitoring should be considered; for instance, PSG is expensive, laborious and inconvenient. Therefore, the development of a simple and convenient device for diagnosing OSA is required. A micromovement sensitive mattress (MSM) is a novel sleep-monitoring system that was first developed by the Institute of Aviation Medicine of the Chinese Air Force (13). The MSM monitoring system features sensors in a sheet, and can detect the pressure of respiratory movements, heart rate and the pressure of body movements. The MSM converts the sensory output into digital signals, and ultimately these may be used to reconstruct sleep breathing patterns. The greatest advantage of this device is that suspected OSA patients do not need to be restrained by attached sensors and consequently experience a more comfortable sleep (13). In Japan, a similar sheet-type portable monitor was produced and demonstrated that the sensitivity and specificity were relatively high (14-16). However, the device requires validation for use in the Chinese Han population.

The aim of the present study was to compare MSM with nocturnal PSG monitoring in order to determine the accuracy and validity of MSM in a clinical environment. 


\section{Subjects and methods}

Study subjects and clinical measurements. The present study enrolled 180 consecutive suspected OSA patients between June 2013 and June 2014 from the Department of Otolaryngology, Shanghai Jiao Tong University Affiliated Sixth People's Hospital (Shanghai, China). All participants had self-reported habitual snoring and excessive daytime sleepiness, with or without nocturnal apnea. The exclusion criteria included: i) Patients with an implanted electronic device; ii) patients who had received or were receiving therapy for OSA; iii) patients with severe systemic diseases; and iv) pregnant women. In addition, patients with severe back pain and spinal deformities that would affect the MSM monitoring application were excluded. Ultimately, 135 Han-Chinese participants (112 males, 23 females; mean age, $44.39 \pm 12.07$ years; mean BMI, $26.92 \pm 3.31 \mathrm{~kg} / \mathrm{m}^{2}$ ) met the criteria and were analyzed.

Height and weight were measured, and the body mass index (BMI) was calculated as BMI $=$ weight $/$ height ${ }^{2}\left(\mathrm{~kg} / \mathrm{m}^{2}\right)$. All subjects underwent both PSG and MSM monitoring. It is worth to note that the PSG device did not interfere with the results of MSM. Written informed consent was obtained from each participant, and the study was approved by the Ethics Committee of Shanghai Jiao Tong University Affiliated Sixth People's Hospital.

MSM structure and measurement. The MSM monitoring system (Rising Sun Co., Ltd, Beijing, China) consisted of a specially-designed sheet, a finger pulse oxygen saturation $\left(\mathrm{SpO}_{2}\right)$ detector (Rising Sun Co., Ltd.) and a personal computer. The specially-designed sheet had a length of $195 \mathrm{~cm}$, width of $90 \mathrm{~cm}$ and thickness of $10 \mathrm{~cm}$. Micromovement-sensitive pressure sensors were performed in the hospital and were arranged within the sheet. They were able to detect slight pressure caused by heartbeat, respiration and other body movements. Following collection of aforementioned data during patient sleep for one night, the data were recorded on a memory card, transferred to the personal computer and then analyzed using customized analytical software.

Respiratory efforts and subcortical arousals were used to define apnea and hypopnea. Apnea was defined as a decrease of $\geq 50 \%$ in the amplitude of respiratory movements for $\geq 10 \mathrm{sec}$, Hypopnea was defined as a $\geq 30 \%$ decrease in the amplitude of respiratory movement for $\geq 10 \mathrm{sec}$ and an oxygen desaturation decrease of $\geq 5 \%$. Both apnea and hypopnea were combined with respiratory effort and were required to end with subcortical arousal. The apnea-hypopnea index (AHI) detected with the MSM ( $\mathrm{AHI}_{\mathrm{MSM}}$ ) was calculated using the number of apnea and hypopnea events per hour of sleep (13). The MSM system will continuously record the events of apnea or hypopnea during the night The mean AHI value for the duration of the sleep was calculated after collecting the number of events per hour.

Overnight PSG measurement. Overnight PSG was conducted using standard digital polysomnographic evaluation with an Alice 4 or Alice 5 Diagnostic Sleep system (Philips Respironics Inc., Murrysville, PA, USA), simultaneously with MSM monitoring between 21:00 and 6:00. In addition, an electroencephalogram (C4-A2, C3-A1, O2-A1 and O1-A2; Philips

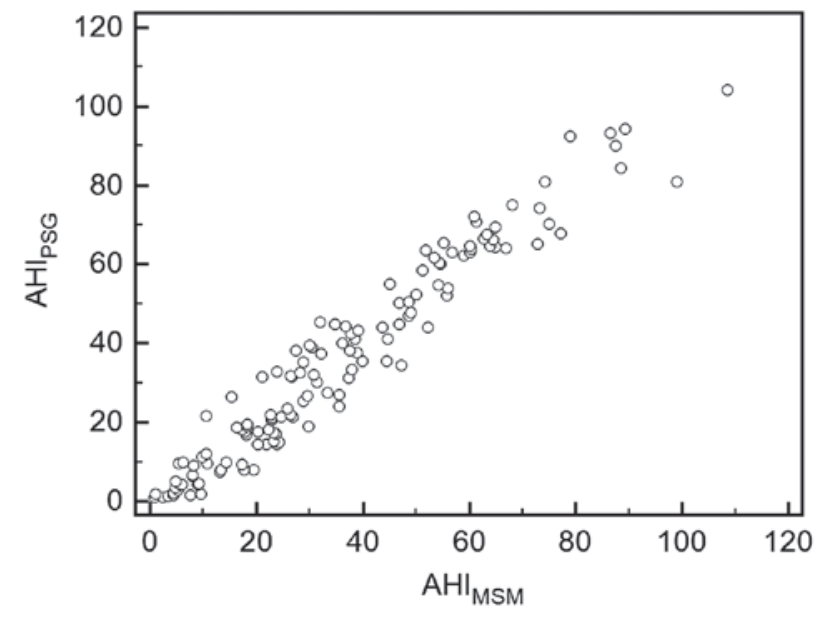

Figure 1. Pearson's correlation between $\mathrm{AHI}_{\mathrm{MSM}}$ and $\mathrm{AHI}_{\mathrm{PSG}}(\mathrm{r}=0.97$, $\mathrm{P}<0.001)$. AHI, apnea-hypopnea index; PSG, polysomnography; MSM, micromovement sensitive mattress.

Respironics, Inc., Murrysville, PA, USA), bilateral electrooculogram, submental and anterior tibial electromyogram, and electrocardiogram (Philips Respironics Inc.,) were recorded with surface electrodes during sleep time. Nasal and oral flow, thoracic and abdominal movement, body posture, snoring and finger $\mathrm{SpO}_{2}$ were also recorded (12). The diagnosis of OSA was assessed according to the AASM criteria, defining apnea events as the cessation of airflow by $\geq 90 \%$ for at least $10 \mathrm{sec}$, and hypopnea events as a reduction in airflow by $\geq 30 \%$ for at least $10 \mathrm{sec}$ and oxygen desaturation of $\geq 4 \%$, which were the criteria for PSG (12). According to AASM criteria, diagnosis and severity of OSA was determined by AHI. Non-OSAHS, mild, moderate and severe OSA were defined as an AHI of $<5$, $5-15,15-30$, and $\geq 30$ events per hour, respectively. AHI monitored by PSG $\left(\mathrm{AHI}_{\mathrm{PSG}}\right)$ was calculated as the total number of apnea and hypopnea events divided by the total number of hours of sleep. According to AASM, AHIPSG of $\geq 5, \geq 15$ and $\geq 30$ represent mild, moderate and severe OSA, respectively. These cut-off values of AHIPSG were used to further classify OSA into different degrees. Therefore, the sensitivity (specificity) for MSM detecting OSA in mild, moderate and severe OSA, respectively, could not be determined. Any mistakes that may have been recorded in the MSM and PSG results were amended manually by a technician, who was blinded to the participant information in order to avoid bias.

Statistical analysis. All the data were analyzed using SPSS software (version 19.0; IBM SPSS, Armonk, NY, USA) and MedCalc (version 12.7.3; MedCalc Software bvba, Ostend, Belgium). Values are presented as the mean \pm standard deviation for continuous variables, and as a number or percentage for categorical variables, as appropriate. The correlation between $\mathrm{AHI}_{\mathrm{PSG}}$ and $\mathrm{AHI}_{\mathrm{MSM}}$ was evaluated using Pearson's correlation coefficient. One-sample t-test was used to evaluate the difference between $\mathrm{AHI}_{\mathrm{PSG}}$ and $\mathrm{AHI}_{\mathrm{MSM}}$. Bland-Altman analysis was performed using MedCalc to assess the similarity between $\mathrm{AHI}_{\mathrm{PSG}}$ and $\mathrm{AHI}_{\mathrm{MSM}}$. OSA was diagnosed in patients that have a higher AHI than the cut-off value. Thus, cut-off points of $\mathrm{AHI}_{\mathrm{PSG}}$ were estimated to increase the precision of the $\mathrm{AHI}_{\mathrm{MSM}}$ device using the area under the receiver operating 


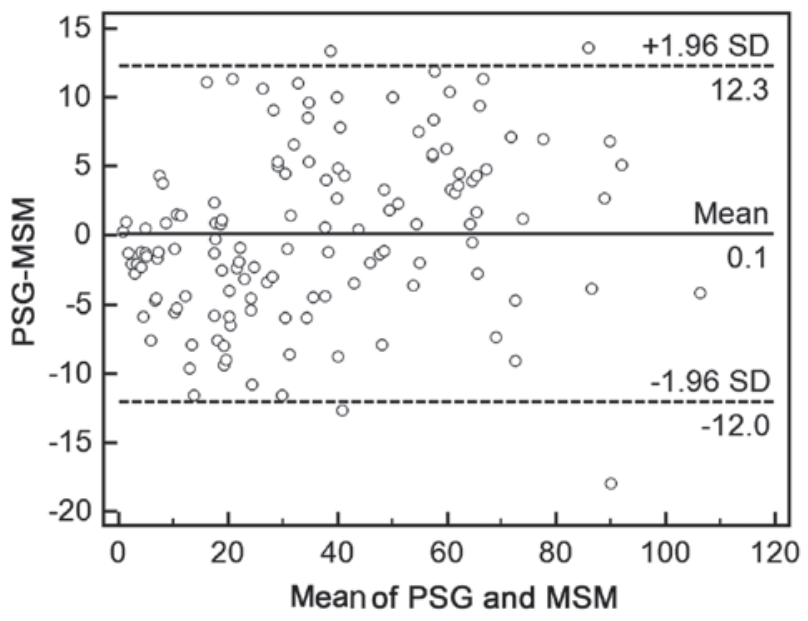

Figure 2. Bland-Altman plot of $\mathrm{AHI}_{\mathrm{MSM}}$ and $\mathrm{AHI}_{\mathrm{PSG}}$ (the upper and lower dotted lines indicate the mean $\pm 1.96 \mathrm{SD}$ ). AHI, apnea-hypopnea index; PSG, polysomnography; MSM, micromovement sensitive mattress; SD, standard deviation.

characteristic (ROC) curves. To assess the predictive performance of the MSM, sensitivity and specificity were also calculated. $\mathrm{P}<0.05$ was considered to indicate a statistically significant difference.

\section{Results}

Patient characteristics. Of the total cohort $(n=135)$, 4 participants were excluded from the study as they did not sleep overnight. The remaining 131 subjects (110 males, 21 females; mean age, $44.33 \pm 11.90$ years; mean BMI, $26.88 \pm 3.24 \mathrm{~kg} / \mathrm{m}^{2}$; mean apnea index, $28.97 \pm 24.25$; mean hypopnea index, $7.47 \pm 7.13$; mean hypopnea events, $53.98 \pm 39.43$; mean obstructive apnea events, $204.33 \pm 176.82$; mean central apnea events, $7.11 \pm 15.31$; mixed apnea events, $8.56 \pm 20.74$; and $\mathrm{SpO}_{2}, 60.63 \pm 29.87 \%$ ). were used in the final statistical analysis. According to electroencephalogram, electrooculogram and electromyogram analyses, the different sleep stages were separated. Mean total sleep time (TST) was $465.98 \pm 64.37 \mathrm{~min}$, mean time in the non-rapid eye movement (NREM) stage was $393.61 \pm 79.42 \mathrm{~min}$, mean time in the rapid eye movement (REM) stage was $69.29 \pm 41.94 \mathrm{~min}$. Percentage of NREM in TST was $84.68 \pm 9.03 \%$, percentage of REM in TST was $15.24 \pm 9.11 \%$, mean time in light sleep was $347.08 \pm 89.75 \mathrm{~min}$, mean time in deep sleep was $41.96 \pm 36.22 \mathrm{~min}$, percentage of light sleep in TST was $74.86 \pm 14.33 \%$, percentage of deep sleep in TST was $9.29 \pm 7.96 \%$. The mean AHI calculated with the PSG and MSM devices were $36.14 \pm 25.50$ and $36.04 \pm 23.89$ events $/ \mathrm{h}$, respectively. In the 14 non-OSA patients, the $\mathrm{AHI}_{\mathrm{PSG}}$ ranged from 1-4.5 events $/ \mathrm{h}$, and the mean $\mathrm{AHI}_{\mathrm{PSG}}$ was $2.61 \pm 1.33$ events $/ \mathrm{h}$. The mean $\mathrm{AHI}_{\mathrm{MSM}}$ was $5.19 \pm 2.86$ events $/ \mathrm{h}$. In the 18 mild OSA patients, the $\mathrm{AHI}_{\mathrm{PSG}}$ ranged from 5.2-14.5 events/h, and the mean $\mathrm{AHI}_{\mathrm{PSG}}$ was $9.65 \pm 2.77$ events $/ \mathrm{h}$. The mean $\mathrm{AHI}_{\mathrm{MSM}}$ was $12.91 \pm 5.98$ events/h. In the 27 moderate OSA patients, the $\mathrm{AHI}_{\mathrm{PSG}}$ ranged from 15.1-27.4 events/h, the mean $\mathrm{AHI}_{\mathrm{PSG}}$ was $20.71 \pm 3.62$ events $/ \mathrm{h}$, the mean $\mathrm{AHI}_{\mathrm{MSM}}$ was $23.46 \pm 6.14$ events/h and in the 72 severe OSA patients, the $\mathrm{AHI}_{\mathrm{PSG}}$ ranged from 30.3-104.2 events/h. The mean $\mathrm{AHI}_{\mathrm{PSG}}$ was $55.07 \pm 18.17$ events $/ \mathrm{h}$ and the mean $\mathrm{AHI}_{\mathrm{MSM}}$ was
A

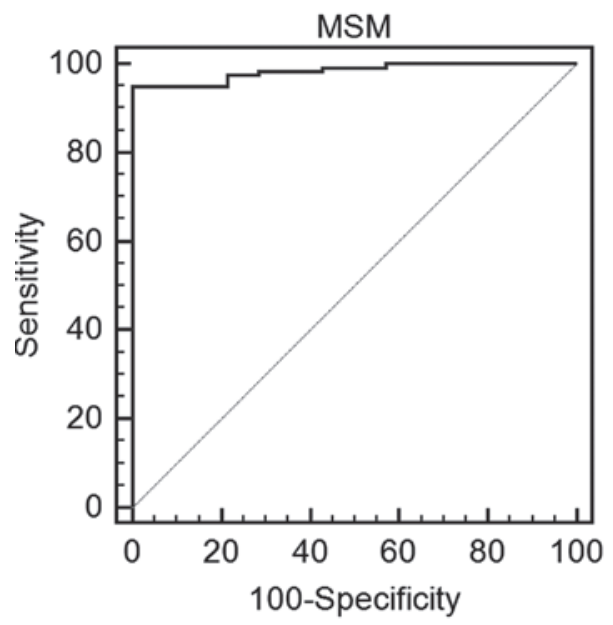

B

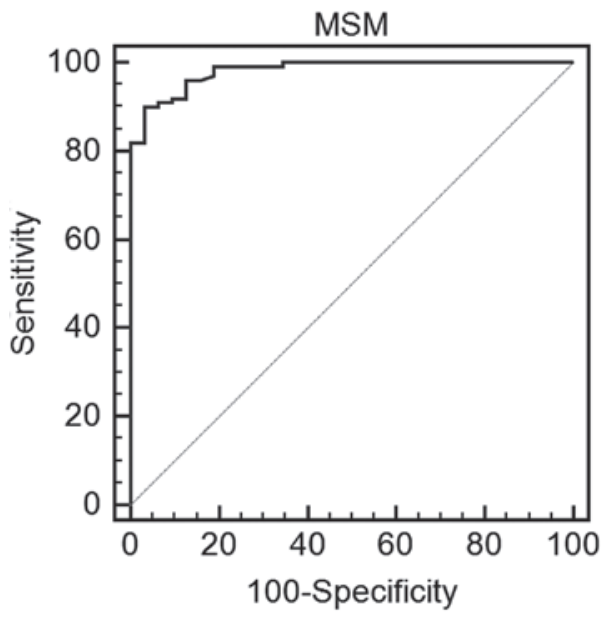

C

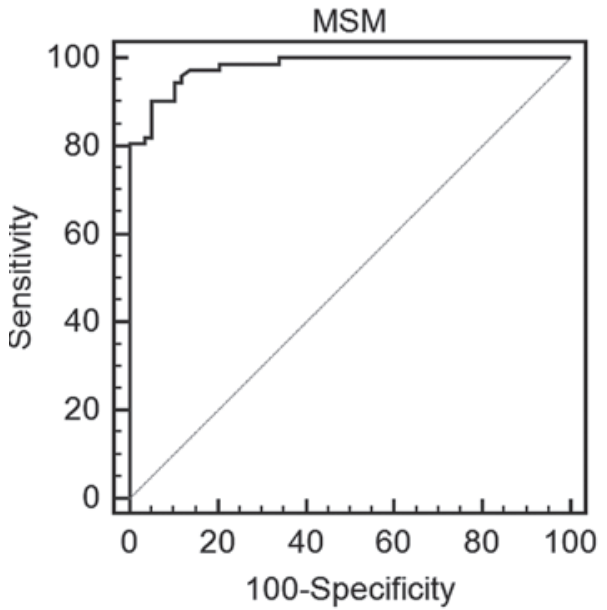

Figure 3. Receiver operating characteristic curves of $\mathrm{AHI}_{\mathrm{MSM}}$ for determining (A) 5 , (B) 15 and (C) 30 events/h with $\mathrm{AHI}_{\mathrm{PSG}}$ : At each $\mathrm{AHI}_{\mathrm{PSG}}$ cut-off value, the sensitivity for detecting $\mathrm{AHI}_{\mathrm{MSM}}$ of $\geq 5, \geq 15$ and $\geq 30$ events/h were $94.9(100 \%), 90.3 \%(94.9 \%)$ and $89.9 \%$ (96.6\%), respectively. MSM, micromovement sensitive mattress; AHI, apnea-hyponea index.

$52.53 \pm 19.01$ events/h. As Bland-Altman analysis revealed there was a good agreement of $97 \%$ between the $\mathrm{AHI}_{\mathrm{PSG}}$ and $\mathrm{AHI}_{\mathrm{MSM}}$ values, thus the diagnosis was consistent between the two methods. According to AHIPSG, 14 participants were non-OSA, 18 were mild OSA, 27 were moderate OSA, and 72 were severe OSA. The mean difference between AHIPSG 
and AHIMSM devices was $0.11 \pm 6.20$, and the difference between them was not statistically significant $(\mathrm{P}=0.84)$.

Comparison of $A H I_{P S G}$ and $A H I_{M S M}$ results. A significant correlation was identified between $\mathrm{AHI}_{\mathrm{PSG}}$ and $\mathrm{AHI}_{\mathrm{MSM}}$ values $(\mathrm{r}=0.97, \mathrm{P}<0.001)$, which are presented as a scatterplot in Fig. 1. Thus, the AHI assessed by the MSM significantly correlated with the AHI simultaneously assessed by PSG. In addition, Bland-Altman analysis revealed a good agreement (97\%) between $\mathrm{AHI}_{\mathrm{PSG}}$ and $\mathrm{AHI}_{\mathrm{MSM}}$ values (Fig. 2). A Bland-Altman plot of AHIMSM versus PSG AHI had only 4 (3\%) outliers for the MSM, and the majority of the AHIs measured by the MSM fell within two standard deviations of the mean PSG value, indicating that differences between AHIPSG and AHIMSM were reasonably tightly distributed.

Sensitivity and specificity of results. ROC curve analysis was also performed to evaluate the clinical use of the MSM as a diagnostic tool for OSA at cut-off values of $\mathrm{AHI}_{\mathrm{PSG}}$ of $\geq 5, \geq 15$ and $\geq 30$ events/h. These values were based on the results of a previous study (12). The results indicated that the percentages of sensitivity (specificity) for detecting an $\mathrm{AHI}_{\mathrm{MSM}}$ of $\geq 5, \geq 15$ and $\geq 30$ events/h were 94.9 (100\%), 89.9 (96.9\%) and 90.3 (94.9\%), respectively. The area under the curve used to differentiate $\mathrm{AHI}_{\mathrm{PSG}}$ of $\geq 5, \geq 15$ and $\geq 30$ events/ $\mathrm{h}$ in the clinical diagnosis of OSA was $0.984,0.982$ and 0.980 , respectively (Fig. 3A-C).

\section{Discussion}

In the present study, the results of Pearson's correlation coefficient analysis revealed that the obtained $\mathrm{AHI}_{\mathrm{MSM}}$ value was correlated with $\mathrm{AHI}_{\mathrm{PSG}}$ value. In addition, Bland-Altman plots provided evidence of the agreement between the results of $\mathrm{AHI}_{\mathrm{MSM}}$ and $\mathrm{AHI}_{\mathrm{PSG}}$. The promising performance of the MSM sleep-monitoring system was also validated compared with PSG monitoring among mild, moderate and severe OSA groups in the Chinese Han population.

As a result of the discomfort imposed upon patients with suspected OSA undergoing PSG monitoring, in addition to being a laborious process for the technician, other simple screening and diagnostic methods have been proposed (17-21). Although mitochondrial DNA mutation appears promising in screening OSA in male patients, the findings require further verification (17). The Berlin questionnaire is also used widely for diagnosis; however, the actual association between the results of the Berlin questionnaire and OSA, is still debated (18). Similarly, the STOPBang questionnaire was recently revealed to have low accuracy, with sensitivities of $81-86 \%$ and specificities of 34-57\% using cut-off values of the PSG respiratory disturbance index of 5,15 and 30 events/h $(19,22)$. Notably, the accuracy of the AHI calculated using snoring analysis compared with the AHI calculated from PSG has been reported to be $96.7,86.7$ and $96.7 \%$ in patients with mild, moderate and severe OSA, respectively (23). Although acoustic analysis has previously shown high agreement with the results of PSG, this technology is sophisticated and requires considerable technical expertise (10). Furthermore, a type 3 portable monitoring (PM) device (Stardust II Sleep Recorder; Philips Respironics, Inc.) has been recommended for use in a clinical environment if the recordings are reviewed manually (21). However, the use of PM also has limitations, since the device is sophisticated and causes patient discomfort, as a finger probe, nasal cannula and belt must be attached to the body (24). In addition, PM is not recommended in suspected OSA patients with comorbid conditions, such as insomnia, depression and mania, which may mask symptoms of OSA (25). Consequently, a simpler and more comfortable portable device should be developed.

A sheet-like sleep-monitoring device, termed the static charge sensitive bed (SCSB), was first used for detecting sleep-associated apnea in 1986 (26). Although SCSB demonstrated a high sensitivity, and was easy to use and relatively inexpensive, it only had a limited capacity for identifying apnea events and was unable to distinguish the type of respiratory event. In Japan, a similar sheet-like sleep-monitoring system, termed SD-101, was previously used to detect suspected OSA (14-16). In the study by Agatsuma et al (14), ROC curve analysis of aa respiratory disturbance index (measured by SD-101, Kentzmedico Co. Ltd, Saitama, Japan) of 14 events/h revealed $89.5 \%$ sensitivity and $85.8 \%$ specificity for identifying OSA. Tsukahara et al (15) calculated AHI using the time in bed, and at a cut-off of 14 events/h, the sensitivity and specificity of detecting an AHI of $\geq 20$ events/h were 90.2 and $90.0 \%$, respectively. Kobayashi et al (16) used the SD-101 with percutaneous oxygen saturation detecting an AHI of $>15$ events/h on PSG with a sensitivity (specificity) of 96.9\% (90.5\%) compared with $87.5 \%(85.7 \%)$, respectively.

The MSM sleep-monitoring system used in the present study is a noninvasive diagnostic device that has been validated in the Chinese Han population. The predominant merits of this MSM sleep-monitoring system are the following: i) It is comfortable and does not disturb sleep; ii) it is appropriate for suspected OSA patients unwilling to undergo PSG with connected sensors; iii) the sensitivity and specificity of the MSM sleep-monitoring device are superior to those of the SD-101 device; and iv) it is equipped with an oxygen detector.

Despite the aforementioned advantages, several limitations of the present study should be addressed. First, the relatively small number of participants may bias the results. Therefore, future research to evaluate the clinical utility of the MSM sleep-monitoring system needs to be performed in multiple sleep centers and communities. Furthermore, the study focused on the Chinese Han population, and thus it is essential to confirm the accuracy of the MSM sleep-monitoring system in other populations. In addition, the diagnostic accuracy of MSM in specific subpopulations, such as in extremely obese individuals and in children, should also be evaluated systematically.

In conclusion, the present study obtained good accuracy for diagnosing OSA with a non-restrictive, non-invasive MSM sleep-monitoring system with relatively high sensitivity and specificity. Further community-based studies with larger sample sizes are warranted to confirm whether the MSM sleep-monitoring system is a suitable substitute for PSG monitoring in diagnosing OSA.

\section{References}

1. Young T, Palta M, Dempsey J, Skatrud J, Weber S and Badr S: The occurrence of sleep-disordered breathing among middle-aged adults. N Engl J Med 328: 1230-1235, 1993.

2. Pepin JL, Borel AL, Tamisier R, Baguet JP, Levy P and Dauvilliers Y: Hypertension and sleep: Overview of a tight relationship. Sleep Med Rev 18: 509-519, 2014. 
3. Aurora RN and Punjabi NM: Obstructive sleep apnoea and type 2 diabetes mellitus: A bidirectional association. Lancet Respir Med 1: 329-338, 2013

4. Ali SS, Oni ET, Warraich HJ, Blaha MJ, Blumenthal RS, Karim A, Shaharyar S, Jamal O, Fialkow J, Cury R, et al: Systematic review on noninvasive assessment of subclinical cardiovascular disease in obstructive sleep apnea: New kid on the block! Sleep Med Rev 18: 379-391, 2014.

5. Marshall NS, Wong KK, Cullen SR, Knuiman MW and Grunstein RR: Sleep apnea and 20-year follow-up for all-cause mortality, stroke, and cancer incidence and mortality in the Busselton Health Study cohort. J Clin Sleep Med 10: 355-362, 2014.

6. Hoyos CM, Melehan KL, Liu PY, Grunstein RR and Phillips CL: Does obstructive sleep apnea cause endothelial dysfunction? A critical review of the literature. Sleep Med Rev 20: 15-26, 2015

7. Stevenson MR, Elkington J, Sharwood L, Meuleners L, Ivers R, Boufous S, Williamson A, Haworth N, Quinlan M, Grunstein R, et al: The role of sleepiness, sleep disorders, and the work environment on heavy-vehicle crashes in 2 Australian states. Am J Epidemiol 179: 594-601, 2014.

8. Rodenstein D: Sleep apnea: Traffic and occupational accidents-individual risks, socioeconomic and legal implications. Respiration 78: 241-248, 2009.

9. Yang EH, Hla KM, McHorney CA, Havighurst T, Badr MS and Weber S: Sleep apnea and quality of life. Sleep 23: 535-541, 2000.

10. $\mathrm{Xu} \mathrm{H}$, Zheng $\mathrm{X}$, Jia $\mathrm{W}$ and Yin S: Chromatography/mass spectrometry-based biomarkers in the field of obstructive sleep apnea. Medicine (Baltimore) 94: e1541, 2015.

11. Strollo PJ Jr and Rogers RM: Obstructive sleep apnea N Engl J Med 34: 99-104, 1996.

12. Iber C, Ancoli-Israel S, Chesson AL and Quan SF: The AASM manual for the scoring of sleep and associated events: rules, terminology and technical specifications 1 st edition. American Academy of Sleep Medicine, Westchester, IL, 2007.

13. Zhang QF, Tong YF, She CP, Zhang XR, Song W, Wang LM and Cheng CJ: Comparative analysis of polysomnography and micro-sensitive mattress-sleep monitor used for obstructive sleep apnea hypopnea syndrome. Zhonghua Er Bi Yan Hou Tou Jing Wai Ke Za Zhi 45: 1014-1019, 2010.

14. Agatsuma T, Fujimoto K, Komatsu Y, Urushihata K, Honda T, Tsukahara T and Nomiyama T: A novel device (SD-101) with high accuracy for screening sleep apnoea-hypopnoea syndrome. Respirology 14: 1143-1150, 2009.
15. Tsukahara M,Sakao S,Jujo T, Sakurai T, Terada J,Kunii R, Tanabe N and Tatsumi K: The accuracy and uncertainty of a sheet-type portable monitor as a screening device to identify obstructive sleep apnea-hypopnea syndrome. Intern Med 53: 1307-1313, 2014.

16. Kobayashi M, Namba K, Tsuiki S, Nakamura M, Hayashi M, Mieno Y, Imizu H, Fujita S, Yoshikawa A, Sakakibara H and Inoue Y: Validity of sheet-type portable monitoring device for screening obstructive sleep apnea syndrome. Sleep Breath 17: $589-595,2013$

17. Huang XY, Li H, Xu XM and Wang LX: Mitochondrial DNA mutation screening of male patients with obstructive sleep apnea-hypopnea syndrome. Exp Ther Med 8: 519-524, 2014.

18. Margallo VS, Muxfeldt ES, Guimarães GM and Salles GF: Diagnostic accuracy of the Berlin questionnaire in detecting obstructive sleep apnea in patients with resistant hypertension. J Hypertens 32: 2030-2037, 2014.

19. Ha SC, Lee DL, Abdullah VJ and van Hasselt CA: Evaluation and validation of four translated Chinese questionnaires for obstructive sleep apnea patients in Hong Kong. Sleep Breath 18: 715-721, 2014.

20. Xu H, Song W, Yi H, Hou L, Zhang C, Chen B, Chen Y and Yin S Nocturnal snoring sound analysis in the diagnosis of obstructive sleep apnea in the Chinese Han population. Sleep Breath 19: 599-605, 2015.

21. Santos-Silva R, Sartori DE, Truksinas V, Truksinas E, Alonso FF, Tufik S and Bittencourt LR: Validation of a portable monitoring system for the diagnosis of obstructive sleep apnea syndrome. Sleep 32: 629-636, 2009.

22. Nagappa M, Liao P, Wong J, Auckley D, Ramachandran SK, Memtsoudis S, Mokhlesi B and Chung F: Validation of the stop-bang questionnaire as a screening tool for obstructive sleep apnea among different populations: A Systematic review and meta-analysis. PLoS One 10: e0143697, 2015.

23. Xu H, Song W, Yi H, Hou L, Zhang C, Chen B, Chen Y and Yin S: Nocturnal snoring sound analysis in the diagnosis of obstructive sleep apnea in the Chinese Han population. Sleep Breath 19: 599-605, 2015.

24. Guerrero A, Embid C, Isetta V, Farre R, Duran-Cantolla J, Parra O, Barbé F, Montserrat JM and Masa JF: Management of sleep apnea without high pretest probability or with comorbidities by three nights of portable sleep monitoring. Sleep 37: 1363-1373, 2014.

25. Sunwoo B and Kuna ST: Ambulatory management of patients with sleep apnea: Is there a place for portable monitor testing? Clin Chest Med 31: 299-308, 2010.

26. Salmi T, Partinen M, Hyyppä M and Kronholm E: Automatic analysis of static charge sensitive bed (SCSB) recordings in the evaluation of sleep-related apneas. Acta Neurol Scand 74: 360-364, 1986. 